\title{
A snapshot of the nutritional status of Crohn's disease among adolescents in Brazil: a prospective cross-sectional study
}

Camila Ortiz Prospero Cavalcante Costa ${ }^{1}$, Flair José Carrilho ${ }^{1}$, Valeria Sutti Nunes ${ }^{2}$, Aytan Miranda Sipahi ${ }^{1}$ and Maraci Rodrigues ${ }^{1 *}$

\begin{abstract}
Background: The relationship between nutrition and Crohn's disease (CD) is complex and involves several therapeutic possibilities including: nutrition treatment for malnourished patients, optimization of growth and development, prevention of osteoporosis, first-line therapy for active disease, and maintenance of disease remission. In children and adolescents with $C D$, malnutrition is a common problem that adversely affects the prognosis. In at-risk adolescent CD patients, it is important to assess body composition, food intake, energy expenditure, nutrient balance and serum levels of nutrients before planning interventions for this population. The aim of this study was to provide a snapshot of the nutritional status of adolescents with CD in Brazil.

Methods: We prospectively selected 22 patients with mildly to moderately active CD, 29 patients with inactive CD and 35 controls (first-degree relatives of and in the same age bracket as the CD patients). The age range of participants was between 13.2 and 19.4 years old. We collected anthropometric data including weight, height, and body mass index (BMI), which were expressed as Z scores: weight-for-age, height-for-age and BMI-for-age, respectively, as well as using bioimpedance to determine body composition and assessing the Tanner stage. We also assessed macronutrients and micronutrients (serum levels and dietary intake of both). We used the chi-square test to determine whether any of the studied variables were associated with inactive or active CD. The level of significance was set at $5 \%$ $(p<0.05)$. We have written informed parental consent for participation for any minors and written informed consent for any participants that were adults.
\end{abstract}

Results: The mean values for lean body mass, Tanner stage, height-for-age $Z$ score and BMl-for-age $Z$ score were lower in the active CD group than in the inactive CD and control groups ( $p<0.05$ for both). Compared with the controls, the $\mathrm{CD}$ patients showed significant differences in terms of the quality of dietary intake (particularly in caloric intake, dietary protein intake, dietary fiber intake, and micronutrient intake), which were reflected in the serum levels of nutrients, mainly vitamins $\mathrm{A}$ and $\mathrm{E}(p<0.05)$.

Conclusions: Adolescents with CD (including those with mildly to moderately active or inactive disease) have a nutritional risk, which makes it important to conduct nutritional assessments in such patients.

Keywords: Crohn's disease, Adolescence, Nutritional assessment

\footnotetext{
* Correspondence: maraci@uol.com.br

'Department of Gastroenterology, University of Sao Paulo School of Medicine Hospital das Clínicas, Av. Dr. Eneas de Carvalho Aguiar 255, 05403-000 Sao Paulo, Brazil

Full list of author information is available at the end of the article
} 


\section{Background}

In approximately $25-30 \%$ of all patients with $C D$, the onset of the disease occurs before 20 years of age. Because the peak onset of pediatric inflammatory bowel disease (IBD) occurs in late adolescence, this event may turn out to be a potent influence on puberty and growth development [1-3].

In this setting, the most specific complication of pediatric $\mathrm{CD}$ is growth deficit, which is caused by a combination of inadequate caloric intake, increased loss of calories and persistent active inflammation of the intestinal mucosa [4]. In such patients, excessive weight loss and malnutrition result in abnormal anthropometric measurements. Therefore, in patients with IBD (principally in those with $\mathrm{CD}$ ), body mass indices (BMIs) and weights are below normal when compared with reference values or with the values reported for healthy controls $[5,6]$. Particularly at this age, micronutrient deficiencies can influence the progression and clinical outcome of IBD, affecting the immune and antioxidant defense systems, as well as tissue repair, growth, and bone mineralization [7].

The presence of nutritional changes in adolescents with $\mathrm{CD}$ has not always been adequately investigated or given sufficient attention by physicians. Nutritional changes in such patients are directly influenced by the society in which they live and the treatment given. Therefore, data from other countries cannot be extrapolated to Brazil. This, together with the lack of studies examining this issue in the country, motivated us to conduct the present study.

\section{Methods}

The study population consisted of patients with a confirmed diagnosis of $\mathrm{CD}$ [1] undergoing regular treatment at the Clinical Gastroenterology Outpatient Clinic of the University of São Paulo School of Medicine Hospital das Clínicas, located in the city of São Paulo, Brazil, between January 2007 and March 2011. The controls were selected among individuals who were firstdegree relatives of the $\mathrm{CD}$ patients and were in the same age bracket as the latter. The age range of participants was between 13.2 and 19.4 years old. We excluded individuals with intestinal diseases or other diseases affecting the nutritional status. First-degree relatives were used as controls because their cultural and environmental influences were the same as those of their CD counterparts; that is, they had the same dietary habits and lived in the same city. In addition, adherence to the study protocol was higher because the controls were relatives of the $\mathrm{CD}$ patients. For the $\mathrm{CD}$ and control groups, the exclusion criteria were as follows: diagnosis of infection, cancer, severe psychiatric disorder, hypothyroidism, hyperthyroidism, corticosteroid usage in the last two months and pregnancy.
In order to classify $\mathrm{CD}$ in terms of its characteristics and severity, we used the Paris classification (a pediatric modification of the Montreal classification of IBD) [8] and the Pediatric Crohn's Disease Activity Index [9].

The Research Ethics Committee of the University of Sao Paulo School of Medicine Hospital das Clínicas, in the city of Sao Paulo, Brazil, approved the research project.

We have written informed parental consent for participation for any minors and written informed consent for any participants that were adults.

\section{Anthropometry}

Anthropometric variables, such as weight, height, and BMI, as well as bioimpedance, were determined in accordance with standardized methods $[10,11]$. Weight and BMI were expressed as Z scores using the World Health Organization (WHO) program AnthroPlus, version 1.0.4 (http://www.who.int/growthref/tools/en/), and sexual maturation was classified according to the Tanner stage [12], as well as being ranked as pubertal or prepubertal according to the recommendations of the WHO [11].

In the bioimpedance analysis, we used a tetrapolar whole-body analyzer (BIA 310; Biodynamics Corporation, Seattle, WA, USA). According to Woodrow [13], the use of bioimpedance analysis for assessing body composition is a rapid, noninvasive and relatively inexpensive method for estimating the amount of body fat, as well as being a portable and easily applicable method.

\section{Dietary intake}

Dietary intake was assessed by means of a 7-day food diary [14]. The food diary covered the 7 days that preceded the medical consultation, was filled out by patients themselves or their legal guardians, and was reviewed upon delivery in order to minimize procedural errors.

The quantitative variables studied were total energy intake, consumption of macronutrients (carbohydrates, lipids and proteins), and consumption of fiber and micronutrients (vitamins A, B12, C, D, E, folic acid, calcium, iron, potassium, magnesium, sodium and zinc). The calculations were made with the software DietPro, version 5.7i (A. S. Sistemas, Viçosa, Brazil).

To determine the basal metabolic rate for each adolescent, we used the Harris-Benedict equation [15], corrected for gender, age, weight and coefficient of degree of physical activity during the week, deemed as minimal effort for all patients studied. Considering $100 \%$ of dietary reference intakes (DRIs) [16], we ranked total energy intake and micronutrient consumption as below or above the recommended levels. Macronutrient intake was analyzed according to the recommendations of the 
WHO and the United Nations Food and Agriculture Organization, thus categorized as below the lower limit, above the upper limit or within the recommended range [17].

\section{Biochemical parameters}

Blood samples were collected for analysis of serum levels of the following: vitamins A, B12, and E, as well as 25hydroxyvitamin $\mathrm{D}$, folic acid, iron, ionized calcium, zinc, magnesium, potassium, copper, albumin, hemoglobin, cholesterol and triglycerides. The values obtained were analyzed against the reference values adopted by the Central Laboratory of the University of São Paulo School of Medicine Hospital das Clínicas.

\section{Statistical analysis}

We used the chi-square test in order to investigate possible associations between the studied variables and the three groups (inactive $\mathrm{CD}$, active $\mathrm{CD}$, and control). The level of significance was set at $5 \%(p<0.05)$. The food consumption data were processed with the DietPro software, after which they were analyzed with the programs Microsoft Excel, Epi Info, version 6.04, and the Statistical Package for the Social Sciences, version 13.0 (SPSS Inc., Chicago, IL, USA).

\section{Results}

The present study included 86 individuals: 22 in the active CD group (patients with mildly to moderately active $\mathrm{CD}), 29$ in the inactive $\mathrm{CD}$ group, and 35 in the control group. The mean (SD) PCDAI scores (0-100 scale) for patients with inactive, mild, and moderate disease were 7.0 (7.1), 18.3 (10.8), 26.2 (11.4), respectively. The demographic characteristics of the study sample are summarized in Table 1. The clinical characteristics, location and behavior of the CD are summarized in Table 2 . Patients with inactive CD showed an increase of the median duration of the disease, although the difference was not significant. In terms of the location affected, simultaneous involvement of the ileum and colon was most common, followed by isolated involvement of the ileum and isolated involvement of the colon. The most common types of $\mathrm{CD}$ were the non-stricturing non-penetrating type and the penetrating type.

As can be seen in Table 3, the mean Z scores for height-for-age and BMI-for-age were higher in the active $C D$ group than in the inactive $C D$ group, as well as being higher in the inactive $C D$ group than in the control group; the differences among the groups were significant. When compared individually, 2 (9.1 \%) of the patients with active $\mathrm{CD}$ and 1 (3.4\%) of those with inactive $\mathrm{CD}$ had short stature (height-for-age $\mathrm{Z}$ score $<-2)$. In addition, $7(31.8 \%)$ of the patients with active $\mathrm{CD}$ and $3(10.3 \%)$ of those with inactive $\mathrm{CD}$ were malnourished (BMI-for-age $\mathrm{Z}$ score $<-2$ ). The deficit of lean body mass in relation to the fat compartment was more pronounced in the active $C D$ group than in the inactive $\mathrm{CD}$ and control groups. It is of note that some (4.2\%) of the CD patients had a BMI-for-age $\mathrm{Z}$ score $>2$ standard deviations, which characterized them as overweight.

Regarding the Tanner stage of sexual maturation, delayed puberty was observed in $22.7 \%$ of the active CD group patients, compared with only $6.9 \%$ of those in the inactive $\mathrm{CD}$ group; the difference between the two groups was significant. None of the individuals in the control group presented delayed puberty.

The patients that made the greatest use of azathioprine were those with active disease $(95.4 \%)$. At the time of this study, none of the patients were using prednisone. Antibiotics (metronidazole and ciprofloxacin) were used by approximately one-third of the active $\mathrm{CD}$ group patients and half of the inactive $\mathrm{CD}$ group patients. Approximately $23 \%$ of the patients with active CD were using infliximab (Table 4).

Total energy intake was lower than the DRIs in $50 \%$ of the adolescents with active CD, in $3.5 \%$ of those with inactive $\mathrm{CD}$, and in $5.7 \%$ of those in the control group (Fig. 1). Macronutrient intake was found to be below the DRIs in $9.1 \%$ of the patients with active CD and in $5.7 \%$ of the controls; no decrease in carbohydrate intake was observed in the patients with inactive $\mathrm{CD}$. Protein

Table 1 Sociodemographic characteristics of Crohn's disease patients and controls

\begin{tabular}{|c|c|c|c|c|c|c|}
\hline Characteristic & $\begin{array}{l}\text { Active CD (Group I) } \\
(n=22)\end{array}$ & $\begin{array}{l}\text { Inactive CD (Group II) } \\
(n=29)\end{array}$ & $\begin{array}{l}\text { Control (Group III) } \\
(n=35)\end{array}$ & G1 & G2 & G3 \\
\hline Age (years), mean $\pm S D$ & $16.4 \pm 2.5$ & $17.3 \pm 2.19$ & $15.5 \pm 2.31$ & NS & NS & NS \\
\hline \multicolumn{7}{|l|}{ Gender } \\
\hline Male, n (\%) & $12(54.5)$ & $16(55.2)$ & $22(62.8)$ & NS & NS & NS \\
\hline Female, $n(\%)$ & $10(45.5)$ & $13(44.8)$ & $13(37.2)$ & NS & NS & NS \\
\hline \multicolumn{7}{|l|}{ Race } \\
\hline White, $n(\%)$ & $20(90.9)$ & $26(89.6)$ & $32(91.3)$ & NS & NS & NS \\
\hline Non-White, $n(\%)$ & $2(9.1)$ & $3(10.4)$ & $3(8.7)$ & NS & NS & NS \\
\hline
\end{tabular}

CD Crohn's disease, SD: standard deviation, G1 group I vs. group II, G2 group I vs. group III, G3 group II vs. group III, NS not significant 
Table 2 Clinical characteristics, location, and behavior of the disease in patients with active and inactive Crohn's disease, based on Levine et al. [8]

\begin{tabular}{|c|c|c|c|}
\hline & Active CD & Inactive CD & G1 \\
\hline Mean time of disease duration (years) $\pm S D$ & $1.14 \pm 1.06$ & $2.83 \pm 3.34$ & NS \\
\hline Mean age of onset of symptoms (years) $\pm S D$ & $14.4 \pm 3.7$ & $14.1 \pm 3.7$ & NS \\
\hline Mean age at diagnosis (years) $\pm \mathrm{SD}$ & $15.6 \pm 2.38$ & $14.5 \pm 3.66$ & NS \\
\hline Mean PCDAI \pm SD & $18.3 \pm 10.826 .2 \pm 11.4$ & $7.0 \pm 7.1$ & \\
\hline \multicolumn{4}{|l|}{ Location, $n(\%)$} \\
\hline L1(distal 1/3 ileum/limited cecal disease) & $8(36)$ & $11(38)$ & NS \\
\hline L2(colonic) & $3(14)$ & $7(24)$ & NS \\
\hline L3(ileocolonic) & $11(50)$ & $11(38)$ & NS \\
\hline L4 a (upper disease proximal to ligament of Treitz) & 0 & 0 & \\
\hline L4b (upper disease distal to ligament of Treitz and proximal to distal 1/3 ileum & 0 & 0 & \\
\hline \multicolumn{4}{|l|}{ Behavior, $n(\%)$} \\
\hline B1: non-stricturing non-penetrating & $16(73)$ & $25(86)$ & NS \\
\hline B2: structuring & $2(9)$ & 0 & NS \\
\hline B3: penetrating & $4(18)$ & $4(14)$ & NS \\
\hline B2B3: both penetrating and stricturing disease, either at the same or different times & 0 & 0 & \\
\hline p: perianal disease modifier & $2(9)$ & 0 & \\
\hline
\end{tabular}

$C D$ Crohn's disease, SD standard deviation, G1 group I vs. group II

intake was found to be below the DRIs in all three groups, being far lower in the active $\mathrm{CD}$ group than in the inactive $\mathrm{CD}$ and control group $(68.2 \%$ vs. $17.2 \%$ and $14.3 \%$ below the DRIs, respectively). Lipid intake was also found to be below the DRIs, principally in those with active CD (18.2 \% below the DRIs). Dietary fiber intake was low in all groups, being particularly low in the two groups of patients with CD (Fig. 1).

Figure 2 shows the percentage of individuals with a mineral intake below the recommended level. Dietary calcium, iron, potassium, magnesium, selenium, and zinc intake was lowest in the active $\mathrm{CD}$ group, followed by the inactive $\mathrm{CD}$ and control group; the differences among the three groups were statistically significant $(p<0.05$ for all; Fig. 2).

Vitamin intake was lower than recommended in at least $75 \%$ of the patients with active CD (Fig. 3). Vitamin intake was lower in the active $C D$ group than in the inactive $\mathrm{CD}$ group, as well as being lower in the $\mathrm{CD}$ groups than in the control group; the differences were statistically significant $(p<0.05)$.

In comparison with the controls, the patients with $C D$ were deficient in serum ferritin and serum albumin. In addition, serum albumin levels were lowest in the patients in remission; the difference between that group of patients and the remaining two groups showed a trend

Table 3 Anthropometric evaluation of active and inactive Crohn's disease patients and controls

\begin{tabular}{|c|c|c|c|c|c|c|}
\hline Variable & $\begin{array}{l}\text { Active CD } \\
\text { (Group I) } \\
(n=22)\end{array}$ & $\begin{array}{l}\text { Inactive CD } \\
\text { (Group II) } \\
(n=29)\end{array}$ & $\begin{array}{l}\text { Control } \\
\text { (Group III) } \\
(n=35)\end{array}$ & G1 & G2 & G3 \\
\hline \multicolumn{7}{|l|}{ Z score } \\
\hline $\mathrm{Ha}$, mean $\pm \mathrm{SD}$ & $-0.41 \pm 0.90$ & $-0.15 \pm 0.92$ & $0.24 \pm 0.99$ & * & * & * \\
\hline BMla, mean \pm SD & $-1.29 \pm 1.64$ & $0.01 \pm 1.18$ & $0.37 \pm 1.18$ & * & * & * \\
\hline \multicolumn{7}{|l|}{ Bioimpedance analysis } \\
\hline $\mathrm{BF}(\%)$, mean $\pm \mathrm{SD}$ & $23 \pm 0.08$ & $21 \pm 0.05$ & $24 \pm 0.04$ & NS & NS & NS \\
\hline LBM (\%), mean \pm SD & $74 \pm 0.08$ & $79 \pm 0.05$ & $76 \pm 0.04$ & * & * & NS \\
\hline \multicolumn{7}{|l|}{ Tanner staging } \\
\hline Delayed puberty, n (\%) & $5(22.7)$ & $2(6.9)$ & - & * & * & NS \\
\hline
\end{tabular}

$C D$ Crohn's disease, G1 group I vs. group II, G2 group I vs. group III, G3 group II vs. group III, Ha height-for-age, BMla body mass index-for-age, $B F$ body fat, $L B M$ lean body mass, NS not significant; * $p<0.05$ 
Table 4 Distribution of the patients (\%) with active or inactive Crohn's disease according to the drugs that were taken before and during the study

\begin{tabular}{|c|c|c|c|c|c|c|}
\hline \multirow[t]{4}{*}{ Drug } & \multicolumn{2}{|c|}{ Before the study } & \multirow[t]{4}{*}{ G1 } & \multicolumn{2}{|c|}{ During the study } & \multirow[t]{4}{*}{ G1 } \\
\hline & Active CD & Inactive CD & & Active CD & Inactive CD & \\
\hline & $n(\%)$ & $n(\%)$ & & $n(\%)$ & $n(\%)$ & \\
\hline & (Group I) & (Group II) & & (Group I) & (Group II) & \\
\hline Azathioprine & $22(100)$ & $25(86.2)$ & NS & $21(95.4)$ & $18(62.1)$ & $*$ \\
\hline Sulfasalazine & $3(13.6)$ & $3(10.3)$ & NS & $2(9.1)$ & $1(3.5)$ & NS \\
\hline Mesalazine & 20(90.9) & $15(51.7)$ & $*$ & $10(45.4)$ & $16(55.2)$ & NS \\
\hline Ciprofloxacin & $12(54.5)$ & $16(55.2)$ & NS & $3(13.6)$ & $12(41)$ & $*$ \\
\hline Metronidazole & $10(45.4)$ & 11(37.9) & NS & $4(18.2)$ & $2(6.9)$ & NS \\
\hline Infliximab & $8(36.3)$ & $6(20.7)$ & NS & $5(23.1)$ & $5(17.2)$ & NS \\
\hline
\end{tabular}

$C D$ Crohn's disease, G1 group I vs. group II, NS not significant; * $p<0.05$

toward statistical significance $(p<0.05)$. Serum ferritin levels were also lower in the patients in remission with the difference showing a tendency toward statistical significance ( $p<0.05$ vs. the other groups).

Although calcium and magnesium intake was low, in comparison with the DRIs, in all three groups, none of the groups showed serum calcium deficiency or serum magnesium deficiency (Fig. 4). In the present study, the most common dietary mineral deficiency was serum iron deficiency $(p<0.05)$, being more pronounced in the inactive $C D$ group than in the active $C D$ group (in contrast to what was observed for the remaining minerals).

Serum levels of vitamin A and vitamin E were lower in the active $\mathrm{CD}$ group than in the remaining two groups $(p<0.05)$. In contrast, the rate of vitamin B12 deficiency was highest in the inactive $\mathrm{CD}$ group, although the differences among the groups were not significant $(p>0.05)$. None of the groups showed serum vitamin D deficiency or serum folic acid deficiency (Fig. 5).
We did not find significant differences $(p>0.05)$ for the cholesterol and triglycerides levels respectively between the inactive CD $(145.76 \pm 3.65 \mathrm{mg} / \mathrm{dl} ; 94.24 \pm$ $7.47 \mathrm{mg} / \mathrm{dl})$, active CD $(148.27 \pm 2.12 \mathrm{mg} / \mathrm{dl} ; 93.64 \pm$ $5.58 \mathrm{mg} / \mathrm{dl})$ and control group $(147.60 \pm 3.03 \mathrm{mg} / \mathrm{dl}$; $92.23 \pm 6.7 \mathrm{mg} / \mathrm{dl})$. For instance, the hemoglobin results were lowest in the inactive and active CD group (11.86 \pm $0.35 \mathrm{~g} / \mathrm{dl} ; 11.87 \pm 0.39 \mathrm{~g} / \mathrm{dl}$ ) compared to the control group $(12.15 \pm 0.27 \mathrm{~g} / \mathrm{dl})$, but not significantly different.

The CD patients investigated in the present study showed significant changes in body composition and in the quality of dietary intake (particularly in caloric intake, dietary protein intake, dietary fiber intake, and micronutrient intake), which were reflected in the serum levels of nutrients, principally vitamins $\mathrm{A}$ and $\mathrm{E}$.

\section{Discussion}

In the present study, we provided a snapshot of the nutritional status of $\mathrm{CD}$ adolescents in Brazil using instruments

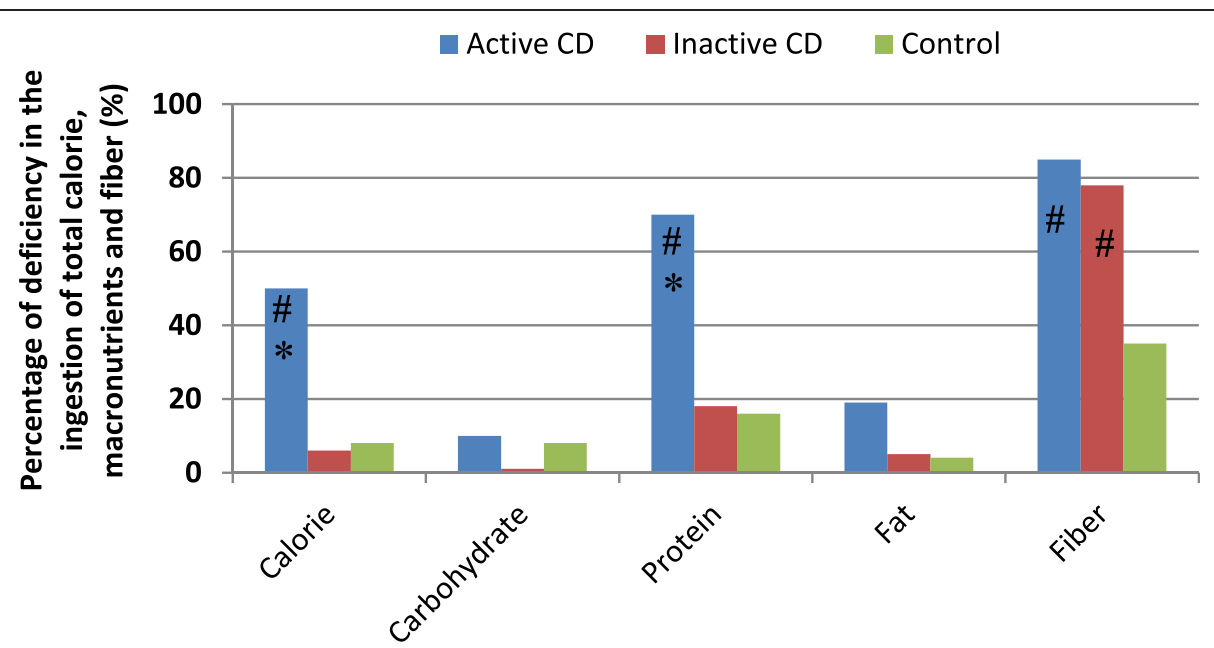

Fig. 1 Deficiency ${ }^{a}$ in the ingestion of total calories, macronutrients and fiber in patients with active DC, patients with inactive DC and control individuals. ${ }^{a}$ Percentange of deficiency is based on the recommendations of the World Health Organization and the United Nations Food and Agriculture Organization [17]. ${ }^{*} p<0.05$ vs. inactive CD group; \# $p<0.05$ vs. control group 


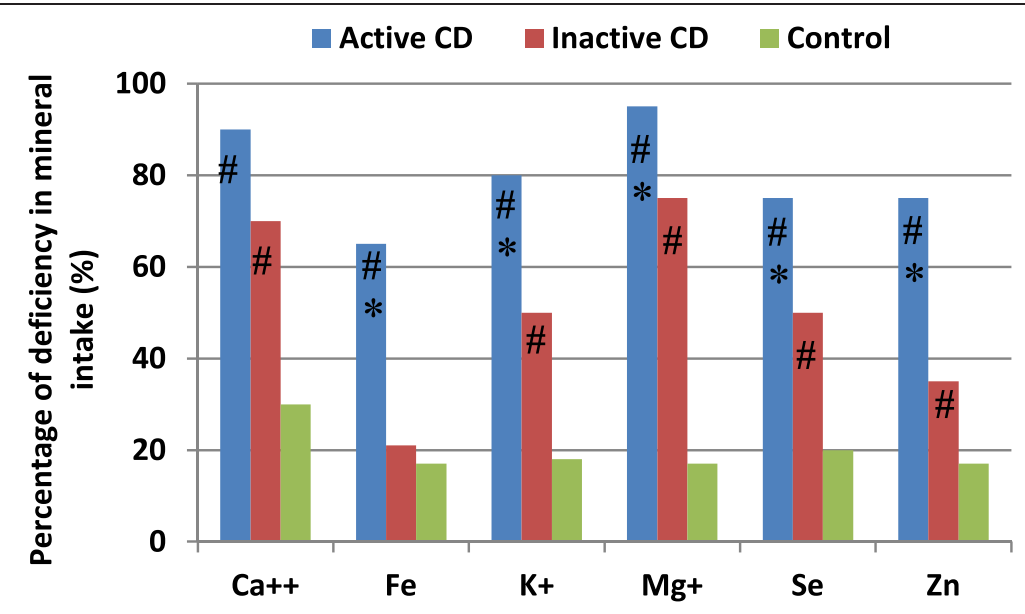

Fig. 2 Percentage of deficiency ${ }^{a}$ in mineral intake in the patients with active DC, patients with inactive DC and control individuals. ${ }^{\text {a Percentange }}$ of deficiency is based on dietary reference intakes [16]. ${ }^{*} p<0.05$ vs. inactive CD group; \# $p<0.05$ vs. control group

and methods that are available at any health facility where such patients might be followed. We found that BMI-forage and height-for-age were significantly lower in the patients with $\mathrm{CD}$ than in the controls, a finding that was consistent with those of Burnham et al. [18], who studied 104 children and young adults with $C D$ evaluated an average of 4 years after the disease had been diagnosed (as was the case in the present study).

In a similar study, Thayu et al. [19] investigated a sample of 78 children and adolescents with $\mathrm{CD}$ and found significant changes in growth, pubertal development, and body composition (in particular, lean body mass loss) at diagnosis in comparison with the control group, although they found no differences between the genders. The deficit of lean body mass persisted throughout the follow-up period.
In the present study, caloric intake was found to be lower than recommended in $50 \%$ of the patients with active $\mathrm{CD}$, in $3.5 \%$ of those with inactive $\mathrm{CD}$, and in $5.7 \%$ of the controls. According to Thomas et al. [20], during disease exacerbation, caloric intake can decrease by $20 \%$ from the recommended intake and the energy deficit can reach $400 \mathrm{kcal}$ per day. Those authors showed that the mean intake of all micronutrients except vitamin B12 was below the DRIs. Likewise, in the present study, the prevalence of micronutrient deficiency was higher among the patients with $\mathrm{CD}$ than among those in the control group. In addition, micronutrient intake was lower in the patients with active $C D$ than in those with inactive $\mathrm{CD}$.

According to Green et al. [21], dietary intake deficiencies are due to major changes in dietary habits occurring

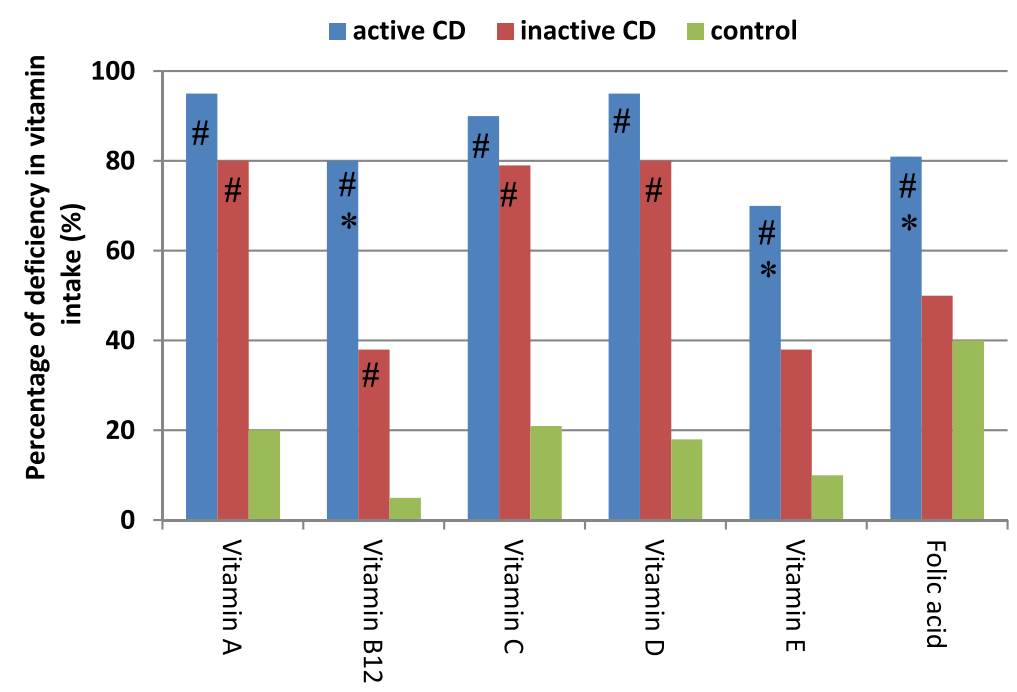

Fig. 3 Percentage of deficiency ${ }^{\mathrm{a}}$ in vitamin intake in the patients with active DC, patients with inactive DC and control individuals. ${ }^{\text {a }}$ Percentage of deficiency is based on dietary reference intakes [16]. ${ }^{*} p<0.05$ vs. inactive CD group; \# $p<0.05$ vs. control group 


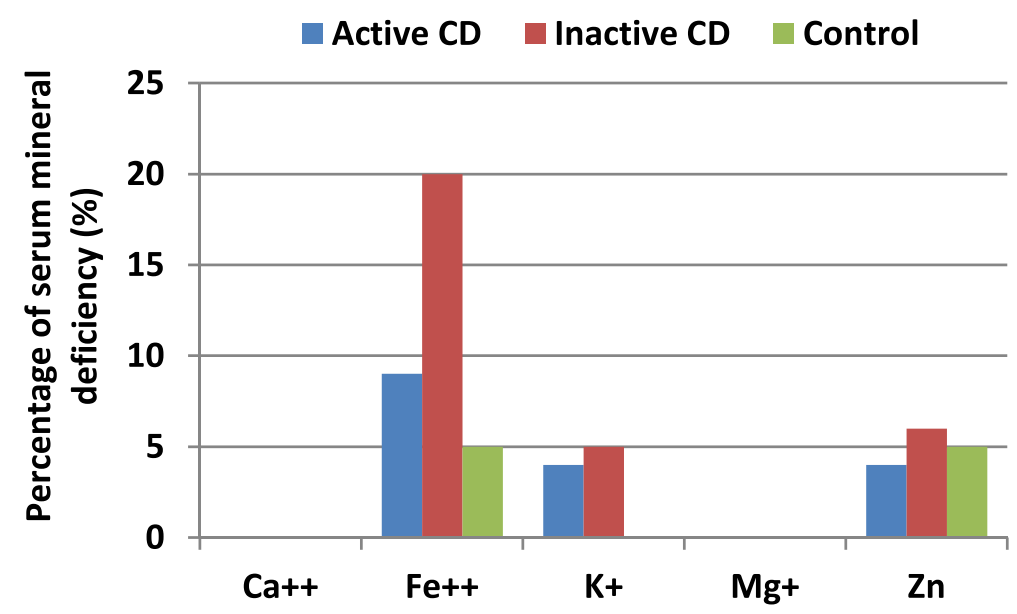

Fig. 4 Percentage of serum mineral deficiency in the patients with active DC, patients with inactive DC and control individuals

after the diagnosis of $\mathrm{CD}$. The authors observed qualitative changes in the dietary habits of pediatric CD patients, who primarily avoided milk and dairy products, as well as fruits and vegetables (because of their high fiber content), mainly for fear of triggering an exacerbation.

Approximately $30 \%$ of the CD patients in our study showed serum iron deficiency, a finding that was consistent with those of at least two studies [22, 23]. What surprised us was the fact that the prevalence of iron deficiency was higher in the patients with inactive $C D$ than in those with active $\mathrm{CD}$. One possible explanation is that many of the patients with active $\mathrm{CD}$ were receiving iron supplements and the hemoglobin was not statistically different between these group. In this regard, the lower iron level observed in the inactive $\mathrm{CD}$ group could be explained with a longer duration of the disease in comparison with the active group, although the values were not statistically different.

We also found that patients with inactive and active $\mathrm{CD}$ were deficient in serum albumin in comparison with the controls, and were lowest in the patients in remission, probably because the parameter used was only PCDAI and no calprotectin or endoscopy parameter was used. In contrast, El-Matary et al. found a significant deficiency of serum albumin in children with active $C D$ using the PCDAI index which correlated with antiSaccharomyces cerevisiae antibody titres [24].

The rates of serum vitamin deficiency were highest for vitamins $A$ and $E$, principally in the patients with active $C D(p<0.05)$. This finding was consistent with Bousvaros et al. [25], who found that vitamin A

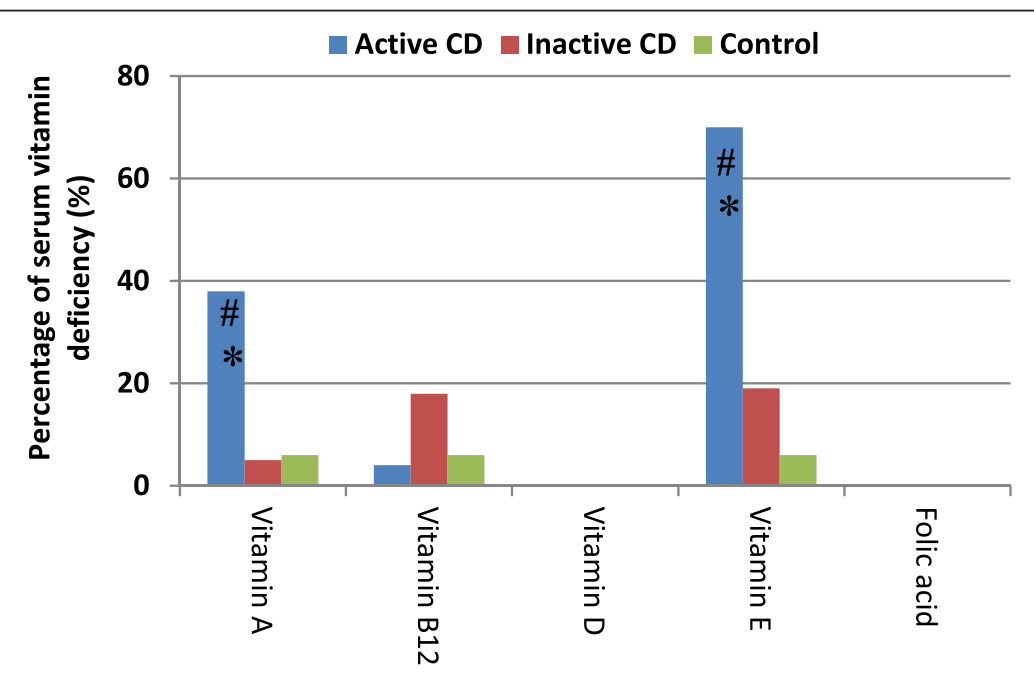

Fig. 5 Percentage of serum vitamin deficiency in the patients with activity $C D$, inactive $C D$ and control group. ${ }^{*} p<0.05$ vs. inactive $C D$ group; \# $p<0.05$ vs. control group 
deficiency and vitamin E deficiency were associated with disease activity. Hoffbrand et al. [26] found changes in plasma levels of antioxidant vitamins in $C D$ patients in comparison with patients with ulcerative colitis and controls.

The limitations of the study included the lack of prealbumin; given its shorter half-life than that of albumin, it is a very good parameter to evaluate nutritional status.

\section{Conclusions}

Adolescents with $\mathrm{CD}$, including those with mildly to moderately active or inactive disease, are at risk for nutritional deficits, which makes it important to conduct nutritional assessments as part of the routine evaluation of such patients, in order to plan nutritional support as part of the overall treatment strategy in this population.

\section{Abbreviations}

BMI: Body mass index; CD: Crohn's disease; DRIs: Dietary reference intakes; IBD: Inflammatory bowel disease.

\section{Competing interests}

The authors declare that they have no competing interest.

\section{Authors' contributions}

COPCC: carried out the analysis, wrote and interpreted the data. FJC: participated in the analysis and interpretation of data. VSN: participated in the analysis and interpretation of data. AMS: participated in the analysis and interpretation of data. MR: coordinated the challenging procedure and designed the study, participated in the analysis and interpretation of data, wrote and finalized the manuscript. All authors read and approved the final manuscript.

\section{Acknowledgments}

The study was supported by the Fundação de Amparo à Pesquisa do Estado de São Paulo (FAPESP, São Paulo Research Foundation).

\section{Author details}

'Department of Gastroenterology, University of Sao Paulo School of Medicine Hospital das Clínicas, Av. Dr. Eneas de Carvalho Aguiar 255, 05403-000 Sao Paulo, Brazil. 'Lipids Laboratory (LIM-10), Endocrinology and Metabolism Division of University of Sao Paulo School of Medicine Hospital das Clinicas, Av.Dr.Eneas de Carvalho Aguiar 255, 05403-000 Sao Paulo, Brazil.

Received: 14 May 2015 Accepted: 30 November 2015

Published online: 08 December 2015

\section{References}

1. Levine A, Koletzko S, Turner D, et al. ESPGHAN revised Porto criteria for the diagnosis of inflammatory bowel disease in children and adolescents. J Pediatr Gastroenterol Nutr. 2014:58(6):795-806.

2. Sauer CG, Kugathasan S. Pediatric inflammatory bowel disease: highlighting pediatric differences in IBD. Gastroenterol Clin North Am. 2009;38(4):611-28.

3. Heuschkel R, Salvestrini C, Beattie RM, et al. Guidelines for management of growth failure in childhood inflammatory bowel disease. Inflamm Bowel Dis. 2008;14(6):839-49.

4. Motil KJ, Grand RJ, Davis-Kraft L, et al. Growth failure in children with inflammatory bowel disease: a prospective study. Gastroenterology. 1993; 105(3):681-91.

5. Markowitz J, Grancher K, Rosa J, et al. Growth failure in pediatric inflammatory bowel disease. J Pediatr Gastroenterol Nutr. 1993;16(4):373-80.

6. Stephens $M$, Batres $L A, N g$ D, et al. Growth failure in the child with inflammatory bowel disease. Semin Gastrointest Dis. 2001;12(4):253-62.

7. Kuroki F, lida M, Tominaga M, et al. Multiple vitamin status in Crohn's disease. Correlation with disease activity. Dig Dis Sci. 1993;38(9):1614-8.
8. Levine A, Griffiths A, Markowitz J, et al. Pediatric modification of the Montreal classification for inflammatory bowel disease: The Paris classification. Inflamm Bowel Dis. 2011;17(6):1314-21.

9. Hyams JS, Ferry GD, Mandel FS, et al. Pediatric Crohn's Disease Activity Index. Development and validation of a pediatric Crohn's disease activity index. J Pediatr Gastroenterol Nutr. 1991;12(4):439-47.

10. Kleinman RE, Baldassano RN, Caplan A, et al. Nutrition support for pediatric patients with inflammatory bowel disease: a clinical report of the North America Society for Pediatric Gastrenterology, Hepatology and Nutrition. J Pediatr Gastroenterol Nutr. 2004;39(1):15-27.

11. de Onis M, Onyango AW, Borghi E, et al. Development of a WHO growth reference for school-aged children and adolescents. Bull World Health Organ. 2007:85(9):660-7.

12. Tanner JM. Growth at adolescence with a general consideration of the effects of hereditary and environmental factors upon growth and maturation from birth to maturity. 2nd ed. Oxford: Blackwell Scientific Publications: 1962

13. Woodrow G. Body composition analysis techniques in adult and pediatric patients: How reliable are they? How useful are they clinically? Perit Dial Int. 2007;27(2):S245-9.

14. Branen L, Fletcher J. Comparison of college students' current eating habits and recollections of their childhood food practices. J Nutr Educ. 1999;31(6): 304-10.

15. Harris JA, Benedict FG. A biometric study of basal metabolism in man. Washington: Carnegie Institution of Washington; 1999.

16. Trumbo P, Schlicker S, Yates AA, et al. Food and Nutrition Board of the Institute of Medicine, The National Academies. Dietary reference intakes for energy, carbohydrate, fiber, fat, fatty acids, cholesterol, protein and amino acids. J Am Diet Assoc. 2002;102(11):1621-30.

17. World Health Organization. Diet, Nutrition and the Prevention of Chronic Diseases. Report of a join WHO/FAU expert consultation. Geneva; 2003 [WHO Technical Report Series,916].

18. Burnham JM, Schults J, Semeao E, et al. Whole body BMC in pediatric Crohn disease: independent effects of altered growth, maturation, and body composition. J Bone Miner Res. 2004;19(12):1961-8.

19. Thayu M, Shults J, Burnham JM, et al. Gender differences in body composition deficits at diagnosis in children and adolescents with Crohn's disease. Inflamm Bowel Dis. 2007;13(9):1121-8.

20. Thomas AG, Taylor F, Miller V. Dietary intake and nutritional treatment in childhood Crohn's disease. J Pediatr Gastroenterol Nutr. 1993;17(1):75-81.

21. Green TJ, Issenman RM, Jacobson K. Patients' diets and preferences in a pediatric population with inflammatory bowel disease. Can J Gastroenterol. 1998;12(8):544-9.

22. Gasche C, Reinisch W, Lochs H. Anemia in Crohn's disease. Importance of of inadequate erythropoietin production and iron deficiency. Dig Dis Sci. 1994; 39:1930-4.

23. Oldenburg B, Koningsberger JC, Berge Henegouwen GP, et al. Iron and inflammatory bowel disease. Aliment Pharmacol Ther. 2001;15:429-38.

24. El-Matary W, Dupuis K, Sokoro A. Anti-Saccharomyces cerevisiae antibody titres correlate well with disease activity in children with Crohn'sdisease. Acta Paediatr. 2015;104(8):827-30.

25. Bousvaros A, Zurakowski D, Duggan C, et al. Vitamins A and E serum levels in children and young adults with inflammatory bowel disease: effects of disease activity. J Pediatr Gastroenterol Nutr. 1998;26(2):129-35.

26. Hoffbrand AV, Stewart JS, Booth CC, et al. Folate deficiency in Crohn's disease: incidence, pathogenesis, and treatment. Br Med J. 1968;13(2):71-5. 